\title{
A rare case of primary parasitic leiomyoma mimicking as ovarian mass: a clinical dilemma
}

\author{
Meenal Sarmalkar*, Arun Nayak, Neha Singh, Madhuri Mehendale, Pratibha Dixit
}

Department of Obstetrics \& Gynecology, Lokmanya Tilak Municipal General Hospital, Sion, Mumbai, Maharashtra, India

Received: 03 December 2015

Accepted: 04 January 2016

\section{*Correspondence:}

Dr. Meenal Sarmalkar,

E-mail: meenal.sarmalkar@gmail.com

Copyright: $\odot$ the author(s), publisher and licensee Medip Academy. This is an open-access article distributed under the terms of the Creative Commons Attribution Non-Commercial License, which permits unrestricted non-commercial use, distribution, and reproduction in any medium, provided the original work is properly cited.

\begin{abstract}
Uterine leiomyomas are the most common benign pelvic tumors in women of reproductive age group. Parasitic or wandering leiomyomas are known for their rarity, atypical clinical presentation \& unusual location, posing challenge in making radiological and clinical diagnosis. More recently, rise in incidence of secondary parasitic leiomyomas has been reported as a complication of laparoscopic myomectomy and morcellations. We report an interesting case of primary parasitic leiomyoma which initially seemed to be a case of ovarian tumor. A 38 year old woman presented with chief complaints of pain in lower abdomen since 4 months with abdominal swelling since 2 months with CT abdomen suggestive of $8.7 \times 5.2 \mathrm{~cm}$ exophytic right ovarian neoplasm with differential diagnosis of broad ligament fibroid in pouch of douglas. She had no previous history of abdominal or laparoscopic myomectomy and no family history of genital tract and breast malignancy. On clinical examination, no mass could be palpated per abdominally but a firm mass of $8 \times 6 \mathrm{~cm}$ size was felt through posterior fornix. Intraoperatively, a solid mass with lobulated surface was noted in pouch of Douglas attached through a thin vascular pedicle to sigmoid colon. Differential diagnosis considering location of tumor and pathological examination included possibility of gastrointestinal stromal tumor or extra-gastrointestinal stromal tumor or wandering leiomyoma. However, on histopathological slide examination (using immunohistochemistry), GIST was ruled out and diagnosis of parasitic fibroid was confirmed. Therefore, while dealing with pelvic tumors with atypical clinical presentation, possibility of these rare tumors have to be kept in mind for appropriate management.
\end{abstract}

Keywords: Parasitic fibroid, Primary parasitic leiomyoma, Rare benign pelvic tumor, Exophytic ovarian neoplasm, Laparoscopic morcellation, Myomectomy

\section{INTRODUCTION}

Uterine leiomyomas are the most common pelvic benign tumours seen in women of reproductive age group with frequently quoted incidence of $50 \%$ found at postmortem examinations. ${ }^{1}$ Parasitic or wandering leiomyomas are very rare extra-uterine benign tumours. As per FIGO classification system, parasitic fibroids have been categorized as Type 8 leiomyomas with no myometrial involvement and uterine attachment. $^{2}$ Parasitic leiomyoma is thought to be originated from a subserous pedunculated myoma which outgrows its blood supply and becomes adherent to omentum or other peritoneal structures like bowel for blood supply. ${ }^{1,3}$ They can be classified further as primary spontaneous and secondary parasitic myomas (which develop after myomectomy procedures). Only few cases of primary parasitic leiomyomas have been reported in literature till date. ${ }^{4}$ In contrast, incidence of secondary parasitic leiomyoma is rising. Recent studies have shown possible association of secondary parasitic leiomyomas with laparoscopic myomectomy or morcellation procedures as its 
complication. $^{5}$ Due to its rarity, atypical clinical presentation and unusual location, these tumours give big challenge to clinicians to reach correct diagnosis preoperatively. Therefore, having clinical suspicion and asking about previous surgical history (especially myomectomy or morcellation) are of utmost importance in making diagnosis. We present an interesting case report where parasitic fibroid was mimicking like an ovarian tumour.

\section{CASE REPORT}

We report a case of primary parasitic leiomyoma in a 38 year old primiparous who attended our gynecology OPD with complaints of pain in lower abdomen since 4 months with abdominal swelling gradually increasing in size since 2 months with outside ultrasound report suggestive of right ovarian cyst measuring $7.6 \times 5.2 \mathrm{~cm}$. There was no history of fever, weight loss and change in appetite, urinary or bowel complaints. There was no history of menstrual irregularity. There was no family history of breast or genital tract malignancy. She gave history of open appendicectomy that was done 25 years back. In her obstetric history, she had one 2 years old living child delivered by cesarean section (no operative record available). On clinical examination, appendicectomy and previous cesarean pfannenstiel scar noted with no obvious swelling or skin changes on inspection. On palpation, abdomen was soft with no palpable mass. There was no guarding, rigidity, tenderness, organomegaly, palpable lymph nodes. On per speculum examination, cervix and vagina were healthy. On bimanual examination, uterus found to be anteverted, mobile and normal in size. A single, firm, non tender mass of $8 \times 6 \mathrm{~cm}$ was felt through posterior fornix. On per rectal examination, same mass was felt without any rectal mucosal infiltration. Ultrasound findings suggested $8.5 \times 5.5 \times 4 \mathrm{~cm}$ mixed echogenic, predominantly hypoechoic, and lobulated lesion in right adnexa with mild to moderate vascularity with few cystic areas, most likely to be broad ligament fibroid or less likely ovarian tumor. CT abdomen was done to confirm USG findings which was suggestive of $8 \times 5 \mathrm{~cm}$ well defined lobulated lesion in POD displacing uterus anteriorly with right ovary not seen separate from it, thus suggestive of right ovarian or paraovarian exophytic neoplasm. In view of inconclusive USG and CT scan findings, MRI pelvis was done. MRI findings suggested presence of a solid lesion with cystic component in POD extending up to L5-S1 level, displacing uterus antero-inferiorly, abutting sigmoid colon $\&$ rectum with the right ovary seen along right lateral aspect, most likely to be benign neoplasm like broad ligament fibroid or solid ovarian neoplasm less likely (Shown in Figure 1).

All preoperative investigations including CA 125 level were within normal range. Patient was posted for exploratory laparotomy with surgical staging with excision of pelvic mass. However, intraoperatively, a firm yellowish lobulated mass of $8 \times 6 \mathrm{~cm}$ was noted in
POD with vascular pedicle attached to anterior surface of sigmoid colon (Shown in Figure 2) with normal uterus, fallopian tubes and ovaries.

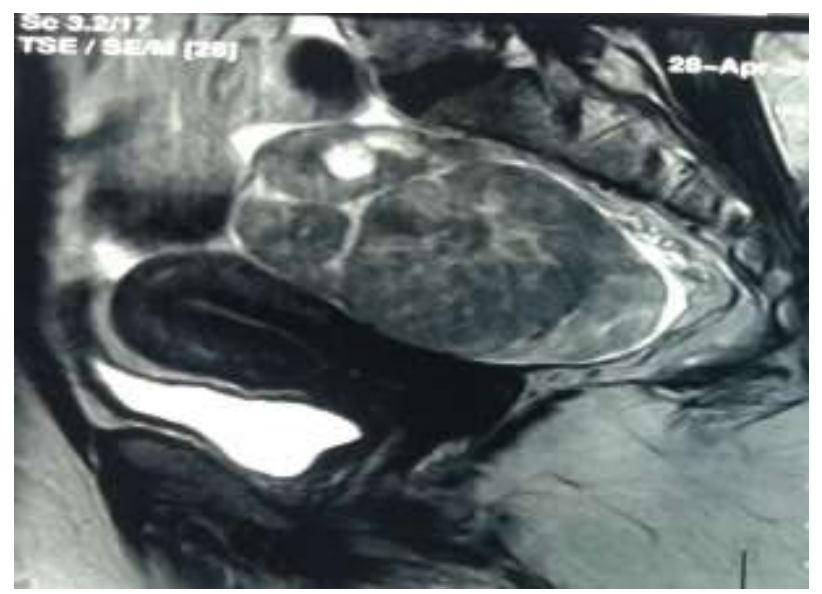

Figure 1: MRI image of pelvic tumour in POD.

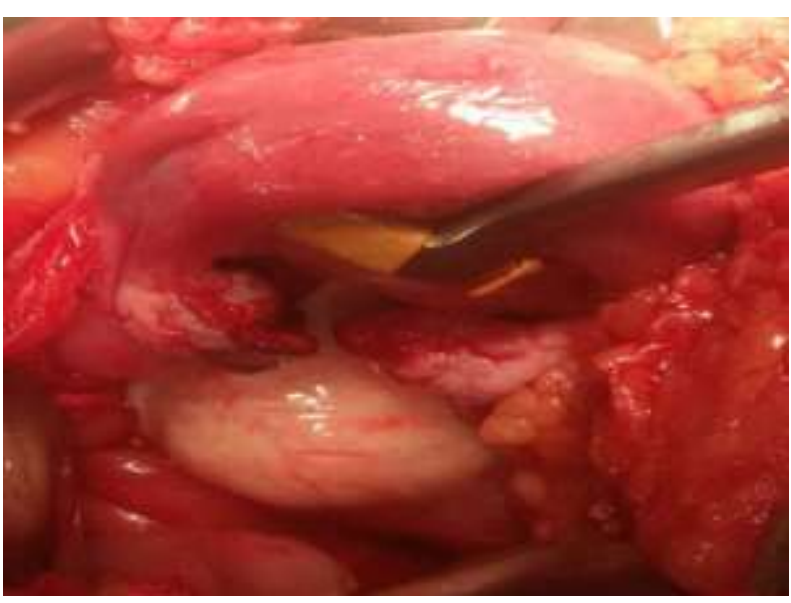

Figure 2: Tumour in POD.

As there was no evidence of involvement of surrounding intraabdominal structures, lymph node dissection was not performed. Feeding vessels were ligated and mass was removed intact (Shown in Figure 3).

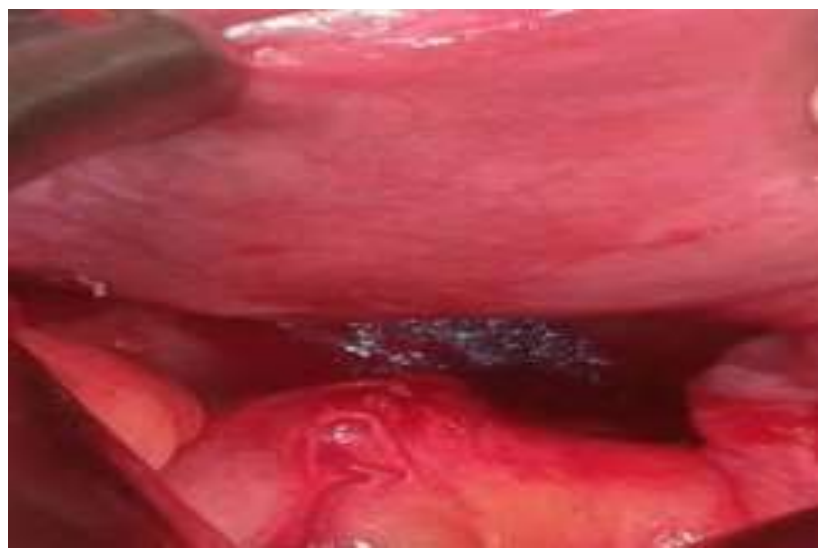

Figure 3: Ligated feeding vessels over surface of sigmoid colon after removal of tumour. 
On gross examination, a globular, firm, grayish white tumor of $7.5 \times 7 \times 8 \mathrm{~cm}$ in size noted and on cut section, whorled appearance was seen (Shown in Figure $4 \& 5$ ).

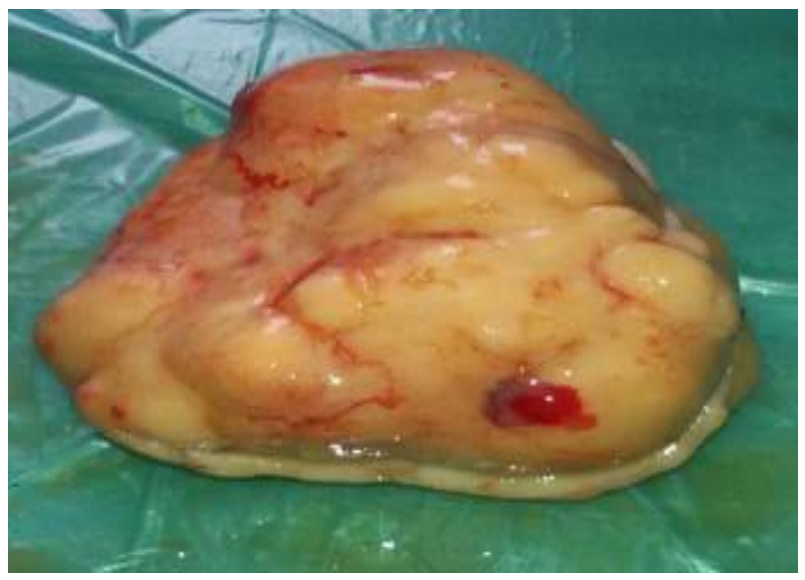

Figure 4: Gross appearance of tumour.

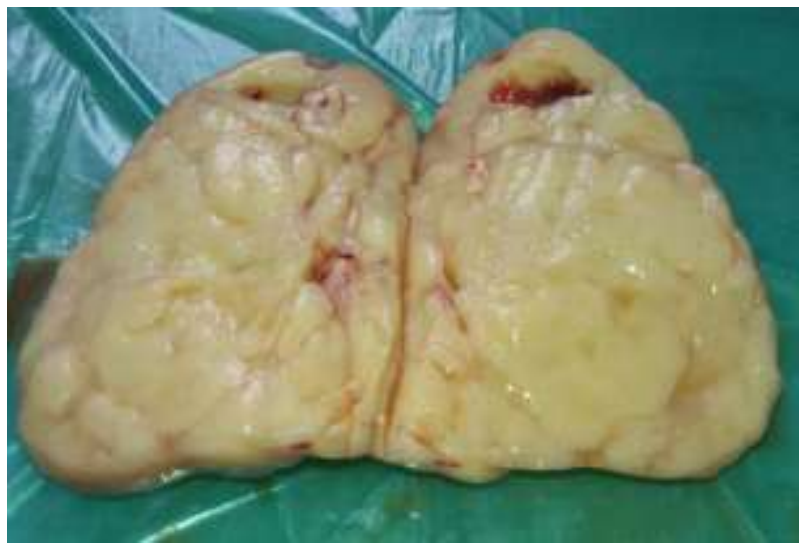

Figure 5: Cut section of tumour.

Microscopic examination confirmed the diagnosis of leiomyoma (Shown in Figure 6). Patient had an uneventful postoperative course and was discharged on day 5 of surgery.

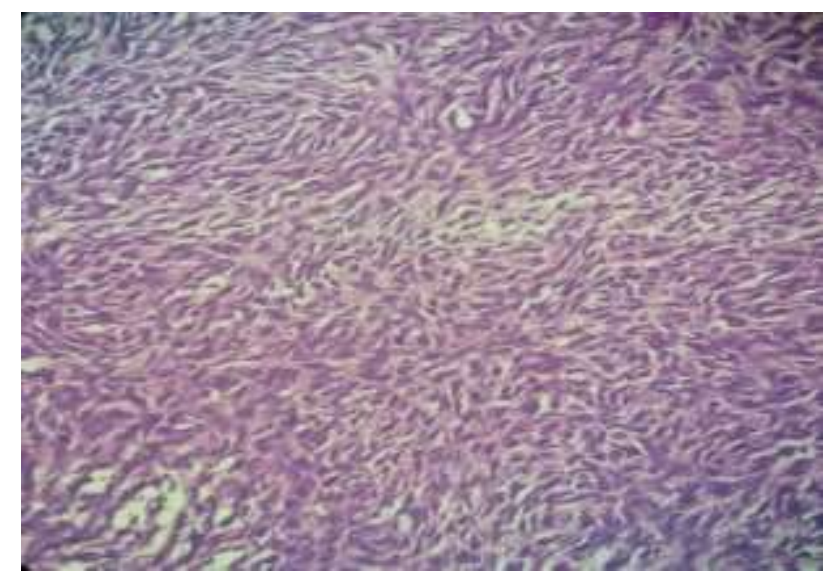

Figure 6: Microscopic appearance of tumour.

\section{DISCUSSION}

Leiomyomas are benign tumours composed of mainly smooth muscles. True incidence of uterine leiomyoma is impossible to determine as many cases remain undetected due to being asymptomatic. Higher incidence has been noted in African American than in Caucasian women. ${ }^{1}$ They have been classified as submucous, intramural, subserosal and transmural fibroids by FIGO classification system. Parasitic fibroids have been categorized as Type 8 leiomyomas with no myometrial involvement and uterine attachment. ${ }^{2}$ Only few cases of primary parasitic leiomyomas have been reported till date. ${ }^{4}$

However, incidence of parasitic leiomyomas is rising nowadays secondary to increased use of laparoscopic morcellation of myomas.

In a retrospective study by Kimberly et al, case records of 12 patients with parasitic myomas were collected. Ten of the 12 cases had history of prior abdominal surgery and eight had prior morcellations procedures. Majority (14 of 15) of myomas were found in pelvis, including two retroperitoneal myomas with one embedded in bladder. Six of 15 myomas were found along the GI tract, and one was found in upper abdomen. This study concluded that more parasitic myomas may be iatrogenically created after surgery, particularly surgery using morcellation techniques and emphasized that surgeons should be aware of the potential for iatrogenic parasitic myoma formation, their likely increasing frequency, and intraoperative precautions to minimize occurrence. ${ }^{5}$

A large retrospective study was done by Gaspare et al to report the development of parasitic myomas after the use of a morcellator over 3 year study period in a tertiary care center. Out of 423 women, in whom electric morcellator was used, four cases were identified to have parasitic myomas with prevalence of $0.9 \%$. This study concluded that laparoscopic myomectomy with the use of a morcellator is associated with an increased risk of developing parasitic myomas. Therefore, a thorough inspection and washing of abdominopelvic cavity should be performed to prevent this rare complication. ${ }^{6}$

Two cases of bowel leiomyomas have also been reported by Pezzuto et al in women who underwent laparoscopic hysterectomy and myomectomy. ${ }^{7}$ On review of literature, only few cases of primary spontaneous parasitic myomas have been reported. In one case, myoma was found in upper abdomen with its vascular pedicle attached to bowel and omentum and in another case, tumour found to be densely adhered to omentum with part of it extending retroperitoneally. ${ }^{4,8}$

Radiological imaging has an important role in making diagnosis. Ultrasonological features of a typical leiomyoma usually include a whorled appearance, with variable echogenicity depending on the extent of degeneration, fibrosis, and calcification. Differential 
diagnosis for parasitic leiomyomas includes ovarian masses (primary tumour or metastatic disease), broad ligament cysts, and lymphadenopathy. Transvaginal US may be of great help in diagnosing broad ligament leiomyomas because it allows clear visual separation of the uterus and ovaries from the mass. MR imaging, with its multiplanar imaging capabilities, also may be extremely useful for differentiating broad ligament leiomyomas from tubo-ovarian masses and from broad ligament cysts and also in differentiating them from solid malignant pelvic tumors. ${ }^{9}$

\section{CONCLUSION}

Parasitic leiomyomas are very rare extra-uterine tumors which are known for their atypical clinical presentation and unusual location, making clinical and radiological diagnosis difficult for clinicians. Thus, they create clinical dilemma due to their tendency to mimic as other pelvic tumours.

Key to appropriate management lies in keeping them in mind as differential diagnosis of various abdominopelvic masses and making best use of imaging techniques in preoperative evaluation. Surgeons must be aware of rare complication (i.e. iatrogenic parasitic leiomyoma) of myomectomy procedures. A thorough inspection and washing of peritoneal cavity must be carried out during morcellation procedures.

\section{Funding: No funding sources} Conflict of interest: None declared Ethical approval: Not required

\section{REFERENCES}

1. Jones HW, Rock JA. Leiomyomata Uteri and Myomectomy. In: Jones HW, eds. Te Linde's Operative Gynecology. 11th ed. Philadelphia, PA: Wolters Kluwer. 2015:658-62.

2. Berek JS. Uterine Fibroids. In: Berek JS, eds. Berek \& Novak's Gynecology. 15th ed. Philadelphia, PA: Lipincott Williams \& Wilkins. 2013:444-5.

3. Robbins SL, Cotran RS, Kumar V. Pathologic basis of disease.3rd ed. Philadelphia, PA: WB Saunders. 1984:1109.

4. Mandal D, Dattaray C, Roy S. Spontaneous Parasitic Leiomyoma: A Rare Clinical Experience. J South Asian Feder Obst Gynae. 2013;5(2):85-6.

5. Kimberly A, Nezhat C. Parasitic Myomas. Obstet Gynecol. 2009;114(3):611-5.

6. Gaspare C, Roberta G, Gloria C, Edgardo S. Parasitic myomas after laparoscopic surgery: an emerging complication in the use of morcellator? Description of four cases. Fertil Steril. 2011;96(2):90-6.

7. Pezzuto A, Serboli G, Ceccaroni M, Ferrari B. Two case reports of bowel leiomyomas and review of literature. Gynecol Endocrinol. 2010;26(12):894-6.

8. Sreelatha S, Kumar A, Nayak V, Punneshetty S. A rare case of primary parasitic leiomyoma. Int J Reprod Contracept Obstet Gynecol. 2013;2(3):422-4.

9. Najila F, Alampady K, Prasad S, David M. Leiomyomas beyond the uterus: unusual locations, rare manifestations. Radiographics. 2008;28:193148.

Cite this article as: Sarmalkar M, Nayak A, Singh N, Mehendale M, Dixit P. A rare case of primary parasitic leiomyoma mimicking as ovarian mass: a clinical dilemma. Int J Reprod Contracept Obstet Gynecol 2016;5:545-8. 